\title{
PRECISE AERIAL IMAGE ORIENTATION USING SAR GROUND CONTROL POINTS FOR MAPPING OF URBAN LANDMARKS
}

\author{
Franz Kurz, Thomas Krauß, Hartmut Runge, Dominik Rosenbaum, Pablo d'Angelo \\ DLR - German Aerospace Center, 82234 Oberpfaffenhofen, \\ (franz.kurz,thomas.krauss,hartmut.runge,dominik.rosenbaum,pablo.angelo)@dlr.de
}

\section{Commission II, WG II/4}

KEY WORDS: Aerial Imagery, Georeferencing Accuracy, Radar-GCPs, Geodetic SAR Processing, Bundle Adjustment, Automatic GCP detection

\begin{abstract}
:
Highly precise ground control points, which are globally available, can be derived from the SAR satellite TerraSAR-X. This opens up many new applications like for example the precise aerial image orientation. In this paper, we propose a method for precise aerial image orientation using spaceborne geodetic Synthetic Aperture Radar Ground Control Points (SAR-GCPs). The precisely oriented aerial imagery can then be used e.g. for mapping of urban landmarks, which support the ego-positioning of autonomous cars. The method for precise image orientation was validated based on two aerial image data sets. SAR-GCPs were measured in images, then the image orientation has been improved by a bundle-adjustment. Results based on check points show, that the accuracy of the image orientation is better than $5 \mathrm{~cm}$ in $\mathrm{X}$ and $\mathrm{Y}$ coordinates.
\end{abstract}

\section{INTRODUCTION}

Ground Control Points from the SAR Satellite TerraSAR-X data have an absolute coordinate accuracy which is comparable to GNSS in-situ measurements. With this new technology, it is no longer necessary to be on-site to obtain high precision ground information. The reference points we use are generally the foot points of poles of lamps or street signs, which can often be clearly identified in both the Radar GCPs and the aerial image.

The assignment in the airborne imagery allows the absolute geo-referencing of the airborne imagery up to the accuracy of one pixel. Using such precisely georeferenced images landmarks suitable for the relative orientation and location of a car may be detected and provided to the autonomous cars in the context of a so called "HD-Map" (Runge et al., 2016, Runge et al., 2018, Richter et al., 2016). Useful landmarks for autonomous driving are for example poles of traffic lights or traffic signs, road markings, trunks of trees or corners of buildings.

In this paper, we propose a method for precise aerial image orientation using spaceborne geodetic Synthetic Aperture Radar Ground Control Points (SAR-GCPs). In section 2, the processing of SAR-GCPs from SAR satellite data is explained. Besides, the usability of the automatically detected poles for the improvement of image orientations will be discussed. In section 3, the process of absolute geo-referencing of the aerial imagery using SAR-GCPs is explained and the results of two flight campaigns are shown. The accuracy of image orientation is validated based on check points, which are measured independently with in-situ GNSS.

\section{GEODETIC SAR POINTS}

\subsection{Deriving Ground-Control-Points from SAR Data}

To remotely measure the position of a radar reflector on the surface of the earth, a radargrammetric method is used in which the exact distances from the satellite to the object on the ground are measured from at least two different angles (satellite orbits). The position of the satellite is known to within a few centimeters, so that the 3D position of the object can be determined by intersection using the radii (Balss et al., 2016).

The high accuracy in the centimeter range is achieved only for so-called "radar point scatterers". These are radar retroreflectors, which are formed by a vertical pole on a flat surface or by a building corner oriented towards the Radar satellite. Figure 1 shows an example of a pole (view from top) measured by TerraSAR-X. Therefore, the method is particularly suitable for urban areas. The high accuracy is further achieved by corrections of the signal path delay caused by ionospheric and atmospheric effects, which are realized in the so-called SAR Geodesy Processor (SGP) of the DLR. Here also a correction of the earth tides takes place. The coordinates are provided in a GNSS-compatible format.

Although two satellite images from different angles are sufficient for determining the coordinates, at least three images are usually used. With the increase in the number of SAR recordings used, the yield of precise ground control points also increases.

The accuracy of the coordinates of individual objects is very different, so we determine the expected accuracy for each individual GCP. It depends on the geometric resolution of the SAR system and on the characteristics of the radar reflector on the ground (the measured object) as well as the local condition in the vicinity of the reflector. The latter determines the so-called "signal to clutter ratio", which is computed for each individual object and is included in the accuracy estimation. A 


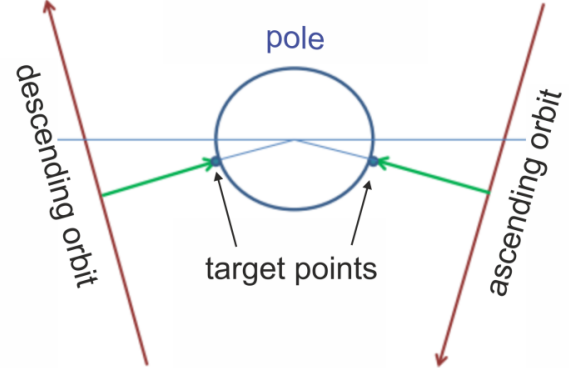

Figure 1. Outline of SAR reflection points at a pole. The reflection point depend on the SAR satellite orbit like ascending or descending of the TerraSAR-X.

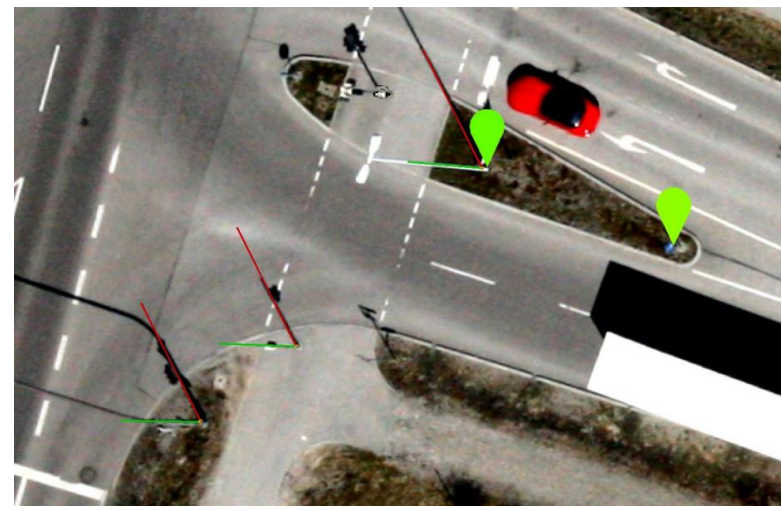

Figure 2. Principle of pole detection - 3K left looking image, red lines: detected shadows, green lines: detected poles, green drops: nearby SAR points

ranking of the expected accuracy of all detected GCPs is output. As proper GCPs only the best objects with an accuracy e. g. better than $10 \mathrm{~cm}$ are in question.

The accuracy of the coordinate determination and the estimation of the error were confirmed by an independent surveying office. The big advantage over GNSS is that one no longer have to be on the spot for the measurement, and for example, a country no longer needs to be entered to determine precise geo-coordinates (Runge et al., 2018). The system is operationally available and used for research and commercial purposes.

\subsection{Extracting Poles from Aerial and Satellite Imagery}

For the improvement of the accuracy of optical or satellite imagery the geodetic SAR points have to be located in the images. Since these points represent the foot points of poles an automatic method for detection of the foot points of poles was developed. Figure 2 shows an example on a road intersection. Filtering out bright narrow objects (the poles, green lines in figure 2) in image looking direction and dark narrow objects (shadows, red lines in figure 2) in shadow direction gives hypotheses of a pole. By intersecting these detected lines the foot points of potential poles can be derived with an accuracy of about half of the ground sampling distance of the images.

Correlating the foot points of the detected poles with nearby geodetic SAR points (the green position marks in figure 2 and applying an outlier detection results in the absolute geographic correction of the image and a list of correlated pole and SAR point locations. Please note, that in the further sections manually measured SAR-GCPs were used.

\section{EXPERIMENTS}

\subsection{Datasets}

Experiments of precise aerial image orientation using SAR ground control points were conducted in two test areas A and B in Germany. Image acquisition flights were performed with the 4k system operated by DLR on a BO105 helicopter (Kurz et al., 2014). It consists of three commercial Canon Cameras mounted together with a GPS receiver and an IMU (inertial measurement unit) on a carrier in such a way one camera looks nadir, one to the left and one to the right. The FOV across/along track of the $4 \mathrm{k}$ system is $70^{\circ}$ resp. $26^{\circ}$.

Dataset A consists of 132 images acquired $400 \mathrm{~m}$ above ground on 2018-10-04 at the DLR site in Oberpfaffenhofen (Germany), which results in a GSD of $5 \mathrm{~cm}$. Only images from the left camera were used, which cover the test area of size $400 \times 400 \mathrm{~m}^{2}$. As the test area is quite small and the camera setup can cover nearly the whole area with one image, the images are highly overlapping with a small baseline. 10 geodetic SAR points were selected covering the whole area for the experiment. The geodetic SAR points were transformed into the ETRF 2000 system according to the epoche of the SAR acquisition date and projected into the UTM system. Additionally, seven check points within the test area were measured with GNSS, corrected with SAPOS and finally transformed into the ETRF system to the epoche of the TerraSAR data acquisition as well.

Dataset B consists of 658 images acquired on 2018-10-02 covering an area of $4 \times 4 \mathrm{~km}^{2}$ over Landsberg am Lech (Germany). The area was acquired with eight flight strips at a flight height of $800 \mathrm{~m}$, which results in a GSD of $10 \mathrm{~cm} .12$ geodetic SAR points were selected covering the whole area for the experiments and divided into three check and nine ground control points. The distribution of check and ground control points for the two test areas is visualized in figure 3 .

The images were further processed to reach the highest georeferencing accuracy. First, the on board measured image positions and attitudes were corrected using the SAPOS service and finally transformed into the ETRF system. Then, a self-calibrating bundle adjustment using automatically matched tie points and manually measured ground control points was performed to improve the image positions and attitudes as well as the interior camera parameters like focal length and sensor distortions (for details see (Kurz et al., 2012)). Finally, all check points were measured manually in the original images and the coordinates of the check points were estimated by spatial reverse intersection. Check points are used to determine the final accuracy of the image orientation by comparing the coordinates with the ground measured in test area A resp. SAR derived coordinates in test area $\mathrm{B}$.

\subsection{Measuring check points and validation of SAR-GCPs}

In this section, the measurement of check points and the validation measurements of geodetic SAR points is described in more detail. As described above, check points are used to determine the accuracy of image orientations, which has been improved using geodetic SAR points in the bundle adjustment. For an independent absolute validation, the check points are neither used in the bundle adjustment nor should depend on the same acquisition system. Thus, for test area A the check points were measured with a terestrial GNSS system, here a Leica 


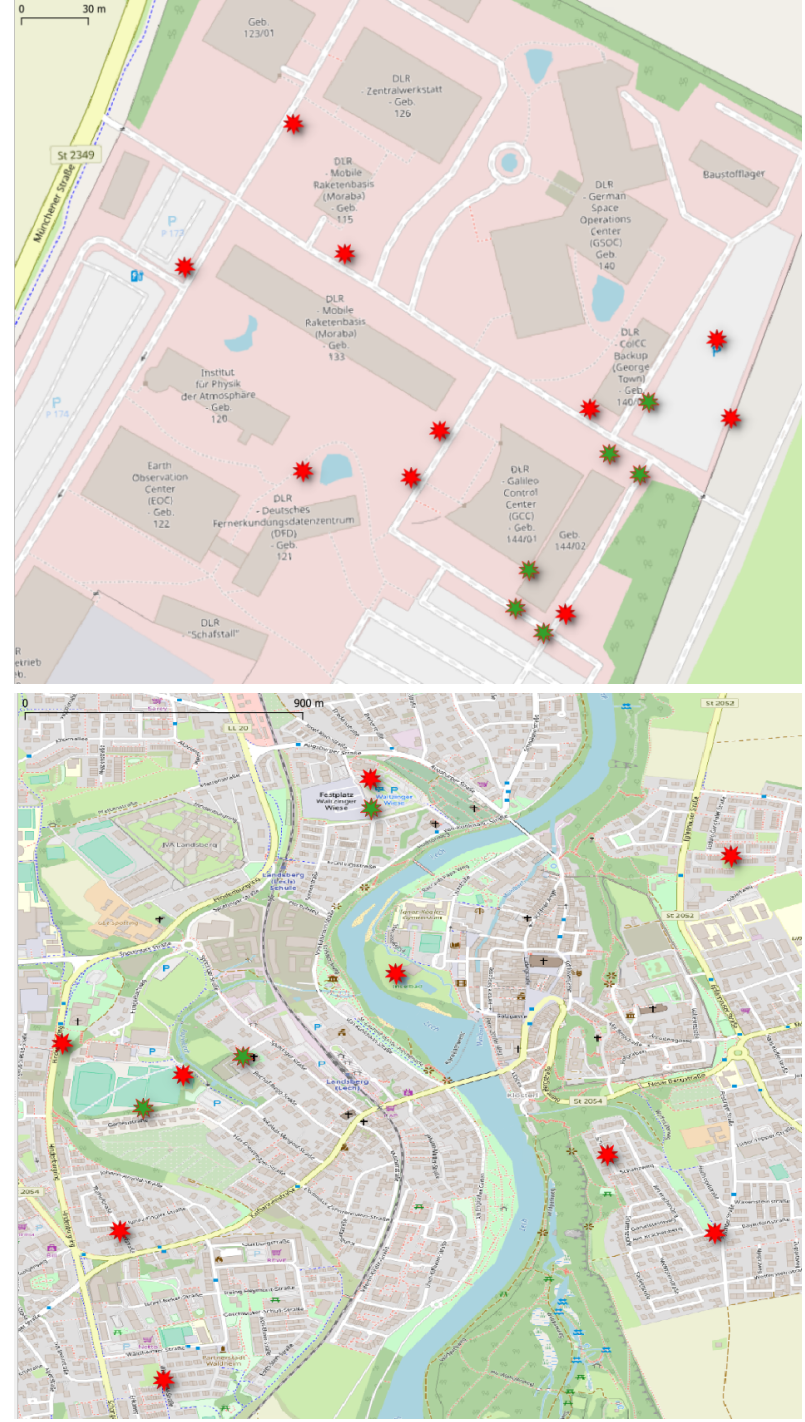

Figure 3. Test area A (Oberpfaffenhofen) and B (Landsberg) with ground control (red stars) and check points (green stars).

GX1230 system. Additionally, a subset of geodetic SAR points was validated with terrestrial GNSS system to check the SAR derived accuracies. The measurement of check points and the validation measurements of geodedtic SAR points in test area A is visualized in figure 4. Two geodetic SAR points \#148 and \#3568 were selected for the validation measurements, which are also used for the improvement of the image orientations.

First, one important issue is the accuracy of the GNSS measurements itself. To reach a sufficient accuracy level, each point was observed at least 10 minutes. The SAPOS service was used to get corrected satellite signals based on a virtual reference station and to transform the points into the ETRF system. Finally, an accuracy level of $1.8-1.9 \mathrm{~cm}$ was reached for each point in test area $\mathrm{A}$ as listed in table 1.

\begin{tabular}{c|c|c|c|c}
\hline & \# pts & $\begin{array}{c}R M S_{X Y Z} \\
\text { @ chkpts }\end{array}$ & \# pts & $\begin{array}{c}R M S_{X Y Z} \\
\text { @SARGCPs }\end{array}$ \\
\hline $\mathrm{A}$ & 6 & $0.018 \mathrm{~m}$ & 2 & $0.019 \mathrm{~m}$ \\
\hline
\end{tabular}

Table 1. Precision of GNSS measurements of selected ground control and check points in test area A

For the GNSS measurements of the geodetic SAR points

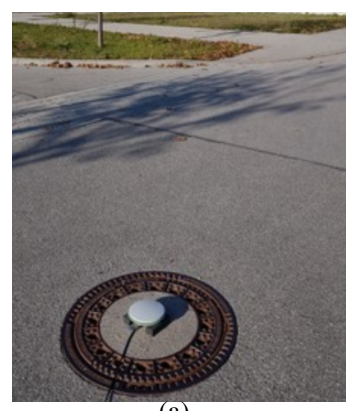

(a)

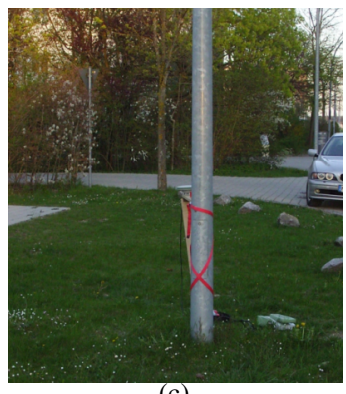

(c)

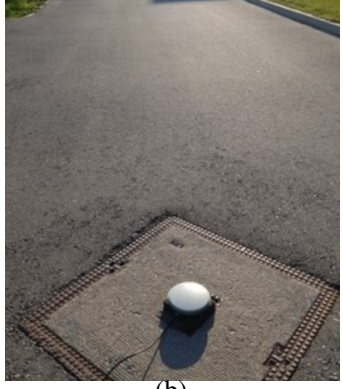

(b)

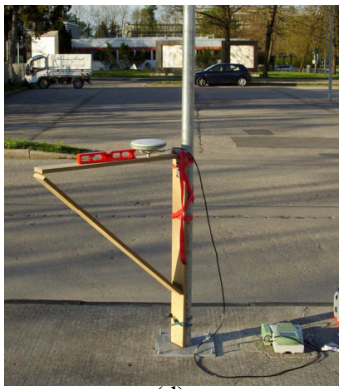

(d)
Figure 4. a) and b) Examples of GNSS measurements at check points in test area A; SAR-GCPs c) \#88 and d) \#3568 during validation with GNSS measurements in test area A.

at poles, a measuring frame construction was necessary to determine the position of the SAR reflection point as illustrated in figure 1. The frame construction is shown in figure $4 \mathrm{c}$ and $\mathrm{d}$. The horizontally aligned bar is measured twice with the GNSS system to get the 3D line. The intersection of the 3D line with the pole minus the relative height provides the coordinates of the SAR reflection point.

\begin{tabular}{c|c|c|c|c|c|c}
\hline \multirow{2}{*}{$\mathrm{pt} \mathrm{nr}$} & \multicolumn{3}{|c|}{ Difference GNSS-SAR } & \multicolumn{3}{c}{ SAR derived $\sigma$} \\
& $\Delta \mathrm{X} \mathrm{m}$ & $\Delta \mathrm{Y} \mathrm{m}$ & $\Delta \mathrm{Z} \mathrm{m}$ & $\sigma_{X} \mathrm{~m}$ & $\sigma_{Y} \mathrm{~m}$ & $\sigma_{Z} \mathrm{~m}$ \\
\hline 148 & 0.050 & -0.044 & 0.017 & 0.060 & 0.011 & 0.025 \\
3568 & 0.126 & 0.012 & 0.029 & 0.069 & 0.296 & 0.345 \\
\hline
\end{tabular}

Table 2. Difference between GNSS positions and geodetic SAR positions of two validation points including the SAR derived precision.

The results of the comparison between GNSS measured and SAR derived coordinates are listed in table 2. The observed coordinate differences at the two points correspond quite well to the predicted precision the SAR points. From this, the conclusion can be drawn that the predicted SAR point accuracy can be trusted. Other validation datasets with more points support this outcome (not yet published).

\subsection{Measuring geodetic SAR points in aerial images}

In this section, the measurement of geodetic SAR points in the images is described in more detail. The most problematic issue in measuring the geodetic SAR points in aerial image is the unique identification of the reflection point of poles. First, most poles are observed in aerial images with a small oblique angle, i.e. the smaller the oblique view the harder the target point can be identified. This effect is illustrated in figure $5 \mathbf{b}$ ), c) and f). In figure 5 a) an example of an oblique observed pole is given with better visibility of the reflection point. In some cases, a street view image can help to identify the SAR reflection point as illustrated in $\mathbf{5} \mathbf{d}$ ). Another feature that hinders a unique recognition is the reduced GSD of the aerial images compared 


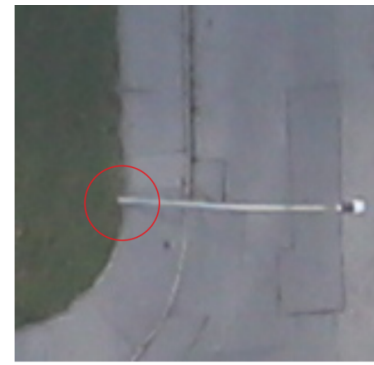

a)

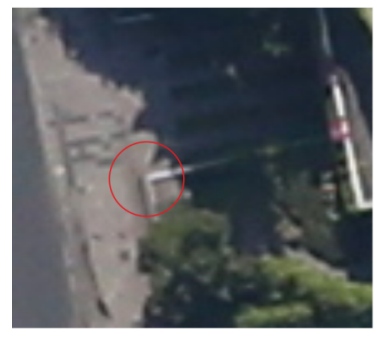

e)

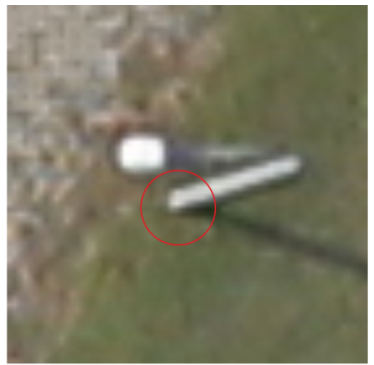

b)

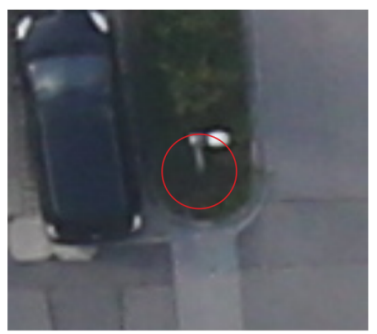

f)

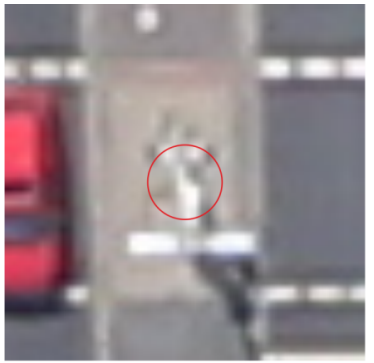

c)

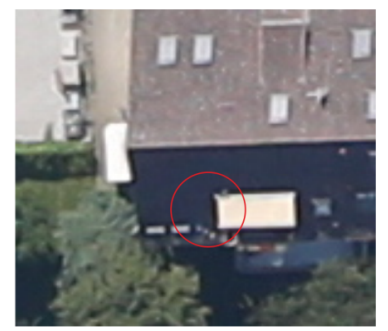

g)

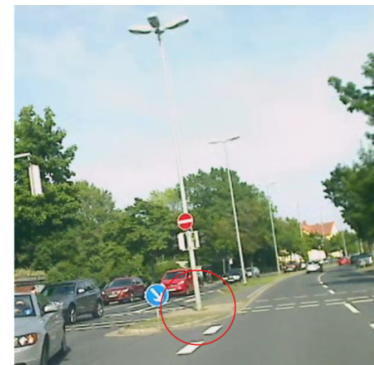

d)

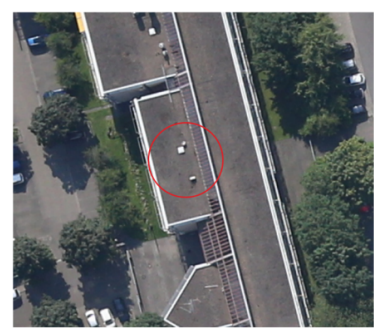

h)

Figure 5. Examples of measuring the SAR-GCPs in aerial images: a),b),e) and f) measurements of pole foot points; c) pole foot point difficult to identify in the aerial image; d) street view image of pole in c) to identify the pole foot point; $\mathbf{g}$ ) and $\mathbf{h}$ ) SAR-GCPs on building roofs difficult to identify.

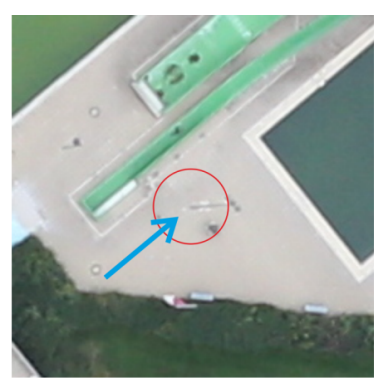

a)

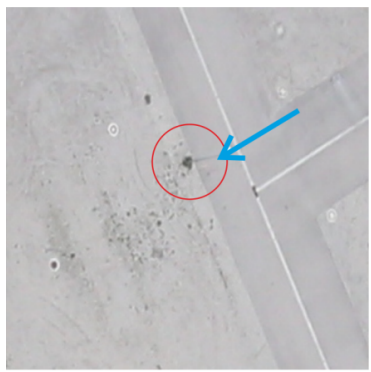

e)

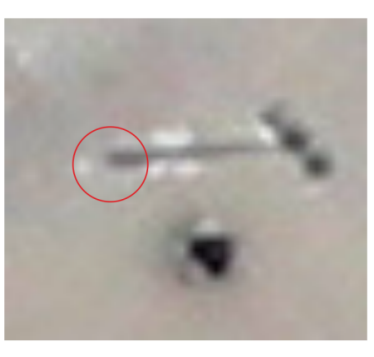

b)

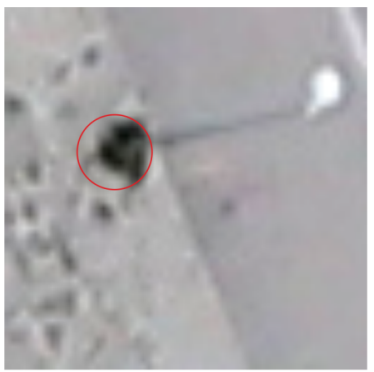

f)

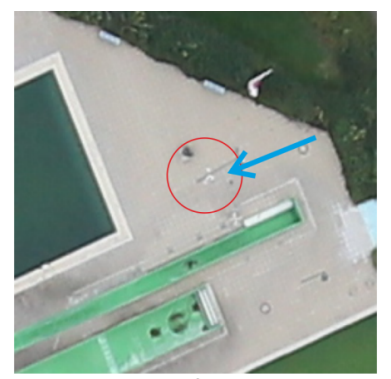

c)

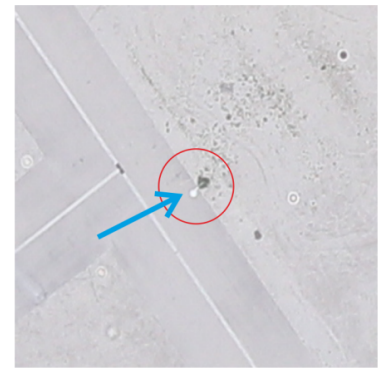

g)

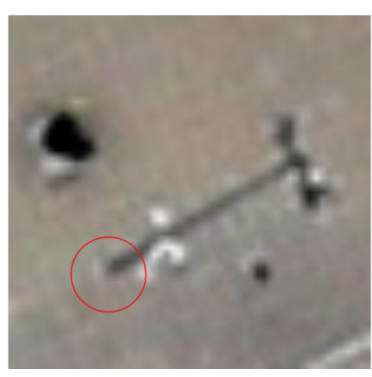

d)

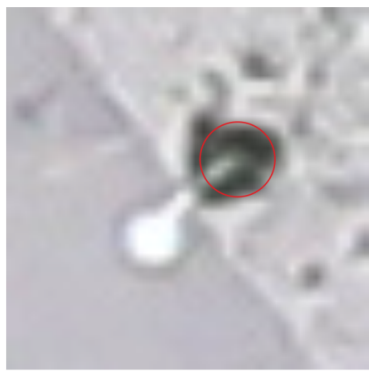

h)

Figure 6. Aerial image samples of SAR-GCPs derived from SAR scenes in one looking direction (blue arrows): a), b), e) and f) poles observed from aerial images in the same looking direction like the SAR satellite; c), d), g) and h) poles observed from aerial images in the opposite looking direction like the SAR satellite. 
to street view. In the examples, based on a GSD of $5 \mathrm{~cm}$ resp. $10 \mathrm{~cm}$ it is hard to identify small objects like small poles. This effect is visualized in figure 5 e) where a pole cannot be distinguished clearly from a wall. Independent of the GSD, it is also difficult to identify SAR reflection points on other objects than poles, e.g. at objects at roofs or buildings like visualized in figure $5 \mathbf{g}$ ) and $\mathbf{h}$ ). These points are filtered out in the process and are not further used.

A specific problem in measuring SAR reflection points in aerial images, is the consideration of the SAR looking direction, when measuring the SAR reflection point. There are two cases, SAR looking direction and viewing direction of the aerial images are the same or opposite. The aerial looking direction depends on the position of the projection center relative to the pole. This effect is illustrated in figure 6. Two poles are imaged from two viewing directions. The SAR looking direction is signalized with a blue arrow. In cases of opposite viewing directions, the reflection point must be guessed in the aerial images, because it is hidden and not visible.

To minimize the error effects caused by low GSD, different looking directions and hard-to-identify SAR reflection points, many SAR points must be measured in the aerial images. It is also necessary, that each ground control and check point must be observed in several images from different viewing directions. Based on the following number of observations, reasonable results in the self-calibrating bundle adjustment were obtained.

- Test area A: 71 measurements of the 16 ground control and check points in 27 (out of 132) images

- Test area B: 60 measurements of the 12 ground control and check points in 46 (out of 658) images

\section{RESULTS}

In this section, the accuracy of the image orientations using SAR-GCPs is presented based on the check points as described above. Base of the validation are the image orientations, which have been improved by a self-calibrating bundle adjustment using SAR-GCPs. Figure 7 show the results of the bundle adjustment in test area $A$. The height of the tie points is analyzed in the left part of the figure, the distribution and residuals of the tie points at the sensor chip level is shown in the right part of the figure. On the one hand, the standard deviation of tie points' height is quite low, on the other hand the observed mean residuals at the sensor chip are below half pixel and therefore sufficiently low.

For the final validation, the coordinates of the check points derived from the aerial images are compared to the coordinates, which are measured with a GNSS system in test area A, or compared with additional, not used geodetic SAR points in test area B. The RMS error of the coordinate differences at the check points are listed in table 3 . In test area A, the RMS error in $\mathrm{X}$ and $\mathrm{Y}$ coordinates lies between $0.04 \mathrm{~m}$ and $0.07 \mathrm{~m}$, whereas the RMS error in $\mathrm{Z}$ shows a higher value at around $0.55 \mathrm{~m}$. The reason for the poor performance in $\mathrm{Z}$ direction is the bad flight configuration with the helicopter in test area $\mathrm{A}$, which produces highly overlapping images with a small base-to-height ratio and therefore produces abrasive cuts with weakly determined $\mathrm{Z}$ values.
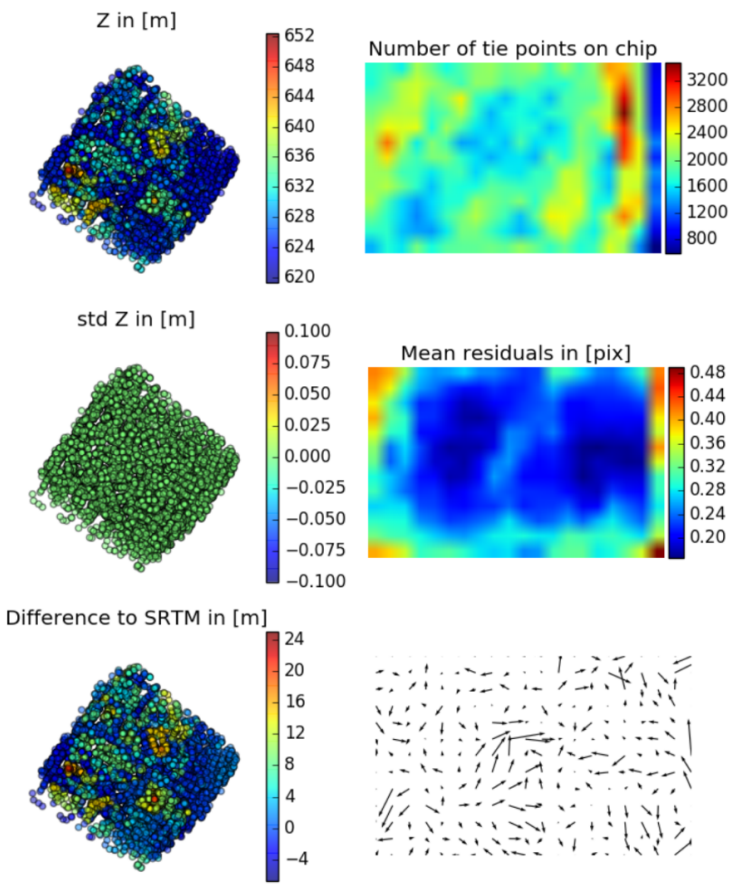

Figure 7. Results of the bundle block adjustment in test area A (counterclockwise from upper left): absolute height of tie points in $\mathrm{m}$, standard deviation of height at tie points, difference of the absolute height to the global SRTM DSM, quiver map of mean residuals at the sensor chip, mean residuals at the sensor chip and distribution of tie points at the sensor chip.

In test area $\mathrm{B}$, the RMS error in $\mathrm{X}$ and $\mathrm{Y}$ lie between $0.03 \mathrm{~m}$ and $0.05 \mathrm{~m}$. The RMS error in $\mathrm{Z}$ is here better than in test area $\mathrm{A}$ because of the better base-to-height ratio of the image bundle.

In both test areas, the RMS X and Y values are quite reasonable and as high as expected. No other systematic effects have been observed, suggesting that the final absolute accuracy is at centimetres level. Besides, the accuracy level of the geodetic SAR points is in the same range of $0.07 \mathrm{~m}$ to $0.15 \mathrm{~m}$, which also hinders to reach an accuracy level in $\mathrm{mm}$ range. The RMS error of all used geodetic SAR points is listed in table 4 .

\begin{tabular}{c|c|c|c|c}
\hline & \# pts & RMS $\Delta \mathrm{X}$ & RMS $\Delta \mathrm{Y}$ & RMS $\Delta \mathrm{Z}$ \\
\hline A & 6 & $0.0734 \mathrm{~m}$ & $0.0396 \mathrm{~m}$ & $0.548 \mathrm{~m}$ \\
$\mathrm{~B}$ & 3 & $0.0259 \mathrm{~m}$ & $0.0513 \mathrm{~m}$ & $0.185 \mathrm{~m}$ \\
\hline
\end{tabular}

Table 3. Final accuracies at check points

\begin{tabular}{c|c|c|c|c}
\hline & \# pts & RMS $\Delta \mathrm{X}$ & RMS $\Delta \mathrm{Y}$ & RMS $\Delta \mathrm{Z}$ \\
\hline A & 10 & $0.098 \mathrm{~m}$ & $0.158 \mathrm{~m}$ & $0.151 \mathrm{~m}$ \\
$\mathrm{~B}$ & 12 & $0.066 \mathrm{~m}$ & $0.111 \mathrm{~m}$ & $0.122 \mathrm{~m}$ \\
\hline
\end{tabular}

Table 4. Precision of SAR ground control and check points derived from SAR measurements.

\section{DISCUSSION}

In this paper we propose a method for precise aerial image orientation using spaceborne geodetic Synthetic Aperture Radar Ground Control Points (SAR-GCPs) with an absolute geolocation accuracy better than $5 \mathrm{~cm}$ in $\mathrm{X}$ and $\mathrm{Y}$ coordinates.

The most important outcome is, that the accuracy level of the image orientations using SAR-GCPs can reach centimeter 
level. This accuracy level seems reasonable, because on the one hand the accuracy of the SAR-GCPs itself have a similar range and on the other hand the identification of the SAR reflection point in the aerial image cannot further be increased based on the configuration in the experimental section. A higher resolution of aerial imagery may improve the identification of the reflection point and thus, may increase the accuracy of the image orientation, provided that the accuracy of the SAR-GCPs itself also further improve.

Future work will be the integration of automatically detected SAR-GCPs into the aerial image orientation process to become independent of manual interactions.

\section{ACKNOWLEDGEMENTS}

The presented technology has been developed in the framework of the project "DriveMark" which was funded by the Helmholtz-Evaluation-Fund. It will be further refined in the cross section project "Digitaler Atlas" of the DLR Traffic Research Branch.

\section{REFERENCES}

Balss, Ulrich, Runge, Hartmut, Suchandt, Steffen, Cong, Xiaoying, 2016. Automated extraction of 3-D ground control points from SAR images - an upcoming novel data product. IGARSS 2016, IEEE Xplore, 5023-5026.

Kurz, F., Rosenbaum, D., Meynberg, O., Mattyus, G., Reinartz, P., 2014. Performance of a real-time sensor and processing system on a helicopter. ISPRS - International Archives of the Photogrammetry, Remote Sensing and Spatial Information Sciences, 189-193.

Kurz, F., Türmer, S., Meynberg, O., Rosenbaum, D., Runge, H., Reinartz, P., Leitloff, J., 2012. Low-cost Systems for real-time Mapping Applications. Photogrammetrie Fernerkundung Geoinformation, 159-176.

Richter, David, Abmayr, Thomas, Runge, Hartmut, 2016. SAR data based landmark navigation for highly precise vehicle localization. MoLaS 2016.

Runge, Hartmut, Balss, Ulrich, Suchandt, Steffen, Klarner, Robert, Cong, Xiaoying, 2016. DriveMark - generation of high resolution road maps with radar satellites. 11th ITS European Congress, ITS Europe, 1-6.

Runge, Hartmut, Balss, Ulrich, Suchandt, Steffen, Krauß, Thomas, Fischer, Peter, Klarner, Robert, Abmayr, Thomas, Wimmer, Michael, Richter, David, 2018. Precise position capture with radar satellites. Munich Satellite Navigation Summit 2018. 